\title{
THE MECHANISM OF THE PULSUS BISFERIENS
}

\author{
BY \\ PETER R. FLEMING \\ From the Cardiac Department, Guy's Hospital
}

Received April 4, 1957

The earliest reference in print to the pulsus bisferiens* is in a Latin edition of Galen's De Pulsibus, published in Paris in 1532. From this, it is clear that debate concerning the nature and significance of this type of pulse dates from the first and second centuries A.D. Galen was insistent that, when two separate impulses are felt in the pulse, it cannot be assumed that two separate pulse waves are present. He stated that the pulsus bisferiens (as distinct from the dicrotic pulse) is characterized by quies media ictus-a central pause in the stroke.

Although clinical observations on the form of the pulse can be found throughout medical literature, it was not until the invention of the sphygmograph by Marey (1860) that more precise information concerning abnormalities in wave-form became available. Mahomed (1872) who used a modification of Marey's instrument, was the first to use the terms percussion wave and tidal wave. These terms are now used to denote the twin peaks of the pulsus bisferiens; the anacrotic pulse is characterized by a small percussion wave followed by an unusually tall and broad tidal wave. Broadbent and Broadbent (1900) were among the first to discuss the pulsus bisferiens in aortic valve disease and mentioned the theory of its causation put forward by d'Espine, who suggested that left ventricular contraction occurred in two phases, producing distinct percussion and tidal waves. Bramwell (1937) was not satisfied with d'Espine's explanation and suggested that the tidal wave represented the summation of outgoing and reflected waves. The slowness of left ventricular ejection in aortic stenosis caused the outgoing wave to be well maintained when the reflected wave returned, the resultant summated tidal wave being very large.

Bramwell's explanation of the genesis of the abnormal pulse forms in aortic valve disease has been quoted widely. An alternative explanation, offered by Katz et al. (1927), has received less attention. They studied the pressure pulses in the left ventricle and aorta in experimental aortic stenosis in dogs and described a sharp negative deflection occurring on the upstroke of the aortic pressure pulse. This they believed to be due to the suction effect of the high-velocity stream of blood (Venturi effect) and regarded it as the analogue of the anacrotic notch in the peripheral pulse.

The introduction of surgical procedures for the relief of aortic stenosis has provided an opportunity of recording pressure pulses in the aorta and left ventricle in cases of aortic stenosis at operation. It is proposed in this paper to study such records in an attempt to elucidate further the mechanism and significance of the abnormal pulse in aortic stenosis-particularly the pulsus bisferiens. None of the previous theories has explained satisfactorily why the pulse associated with pure or dominant aortic stenosis is characterized by a notch on the upstroke and a large tidal wave, whereas if significant aortic regurgitation is superadded, the pulsus bisferiens with equal, and well defined, percussion and tidal waves is found.

\footnotetext{
* The derivation of "bisferiens" is from bis-ferio (= beat twice) and not, as is often thought, from bis-fero (= bear twice).
} 


\section{Material AND Methods}

We have studied 36 cases of aortic stenosis. In some, there was also aortic regurgitation, but in none was it the dominant lesion. Pressure pulses from the left ventricle and aorta were recorded before and after aortic valvotomy; in 29 cases the pressure pulses were recorded simultaneously. Pressures were recorded with 21-gauge needles connected by one metre of hardened plastic tubing to Southern Instruments Minirack electromanometers with a Brush direct-writing recorder.

In cases in which the pressures were recorded simultaneously, the aortic and left ventricular pressure pulses have been redrawn to the same scale and superimposed (Fig. 1 and 2). The gradient across the valve has been measured at intervals throughout systolic ejection and from these

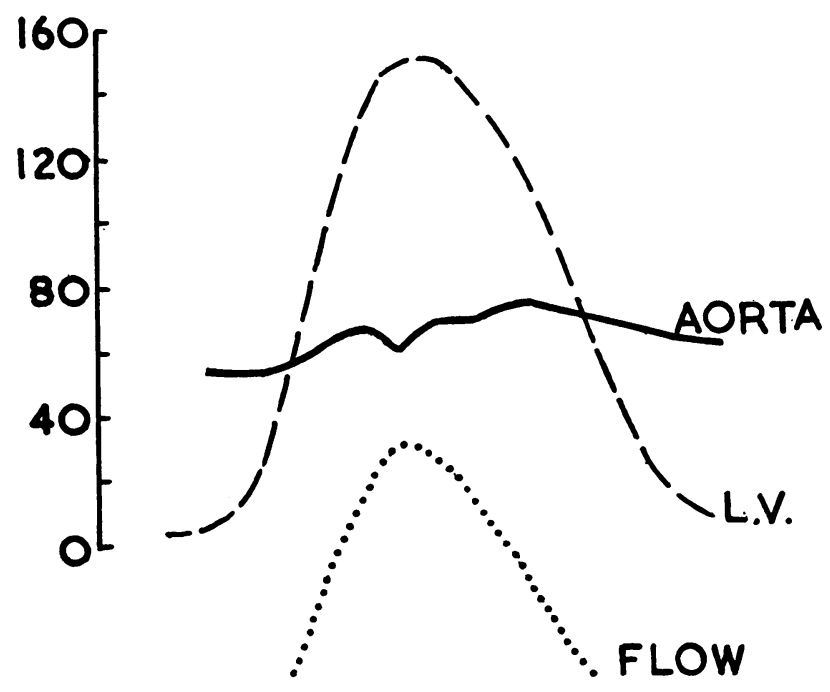

Fig. 1.-Superimposed left ventricular and aortic pressure pulses with flow curve derived from these from a case of aortic stenosis. The apex of the flow curve is synchronous with the aortic anacrotic notch.

measurements a curve representing the changing gradient has been plotted (Fig. 1-lowest curve). The possible error introduced by the measurement of a very high gradient at the nadir of the anacrotic notch was considered and, in practice, measurements were not made at this point. In any case, left ventricular pressure was always so much higher than aortic that quite considerable variations in the form of the aortic pressure pulse had little effect on the form of the gradient curve: the apex of the gradient curve nearly always coincided with the peak of the left ventricular pressure pulse.

The flow through a fixed orifice is directly related to the pressure gradient across the orifice. The gradient curve has, therefore, been used as an approximation to the curve of changing flow through the aortic orifice.

\section{RESULTS}

In 28 of the 36 cases a more or less well-defined notch was present on the upstroke of the aortic pressure pulse; this was sometimes associated with high-frequency vibrations of the type recorded by Katz et al. (1927) (Fig. 3). The upstroke time of the aortic pressure pulse was always prolonged and the aortic systolic peak delayed relative to the left ventricular peak.

After a successful valvotomy (Fig. 4), apart from the fall in systolic pressure gradient across the valve, the anacrotic notch was often situated higher on the upstroke, nearer the aortic systolic peak. As it moved higher, it was usually less well-defined and sometimes could no longer be identified. 


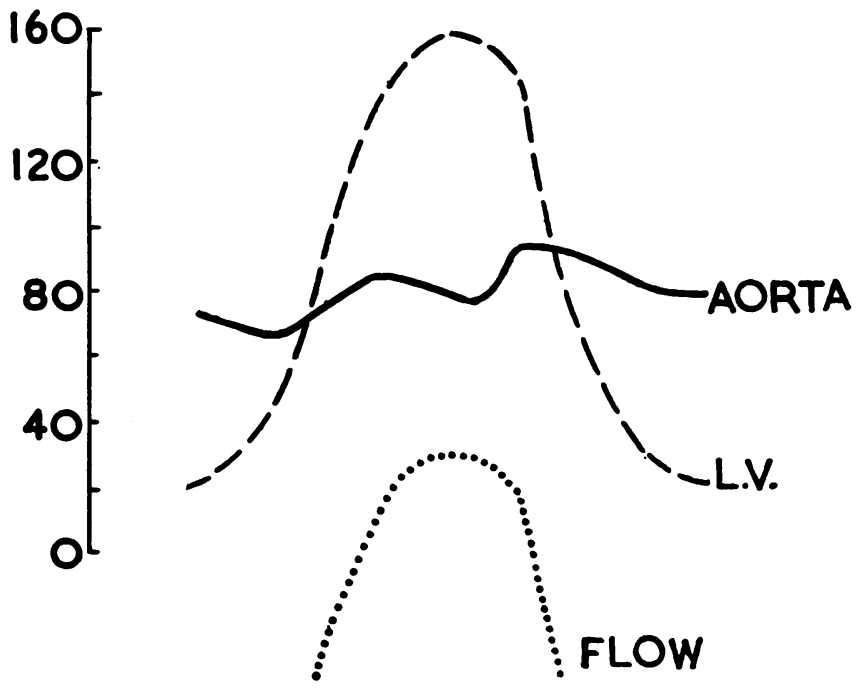

FIG. 2.-Superimposed left ventricular and aortic pressure pulses with flow curve derived from these from a case of aortic stenosis after significant aortic regurgitation had been produced by aortic valvotomy. A deep notch is present very near the summit of the aortic pressure pulse and synchronous with the peak of the flow curve.

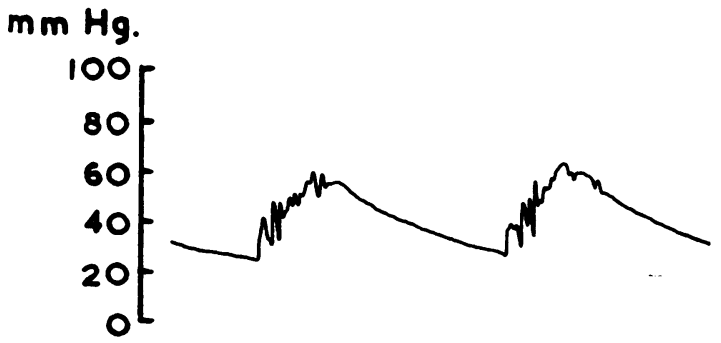

FIG. 3.-Aortic pressure pulse from a case of aortic stenosis, showing anacrotic notch and additional high-frequency vibrations on the upstroke.

These changes were associated with abbreviation of the aortic upstroke time so that it would be more accurate to state that the aortic systolic peak moved towards the anacrotic notch rather than vice versa. In a few cases, although the notch was situated higher on the upstroke after valvotomy, it was deeper than before, producing the appearance of the pulsus bisferiens. In these cases, there was clinical evidence of significant aortic regurgitation after the operation.

In all cases in which superimposed pressure pulses and a derived flow curve were studied, the anacrotic notch coincided precisely with the moment of maximum flow. It has been shown above that this coincidence is most unlikely to be due to over-estimation of the gradient at the nadir of the anacrotic notch.

\section{Discussion}

The coincidence of maximum flow with the anacrotic notch was constant in this series. Study of simultaneous left ventricular and aortic pressure pulses recorded by left atrial and aortic catheterization and published by Wright et al. (1956) shows the same phenomenon. These observations 


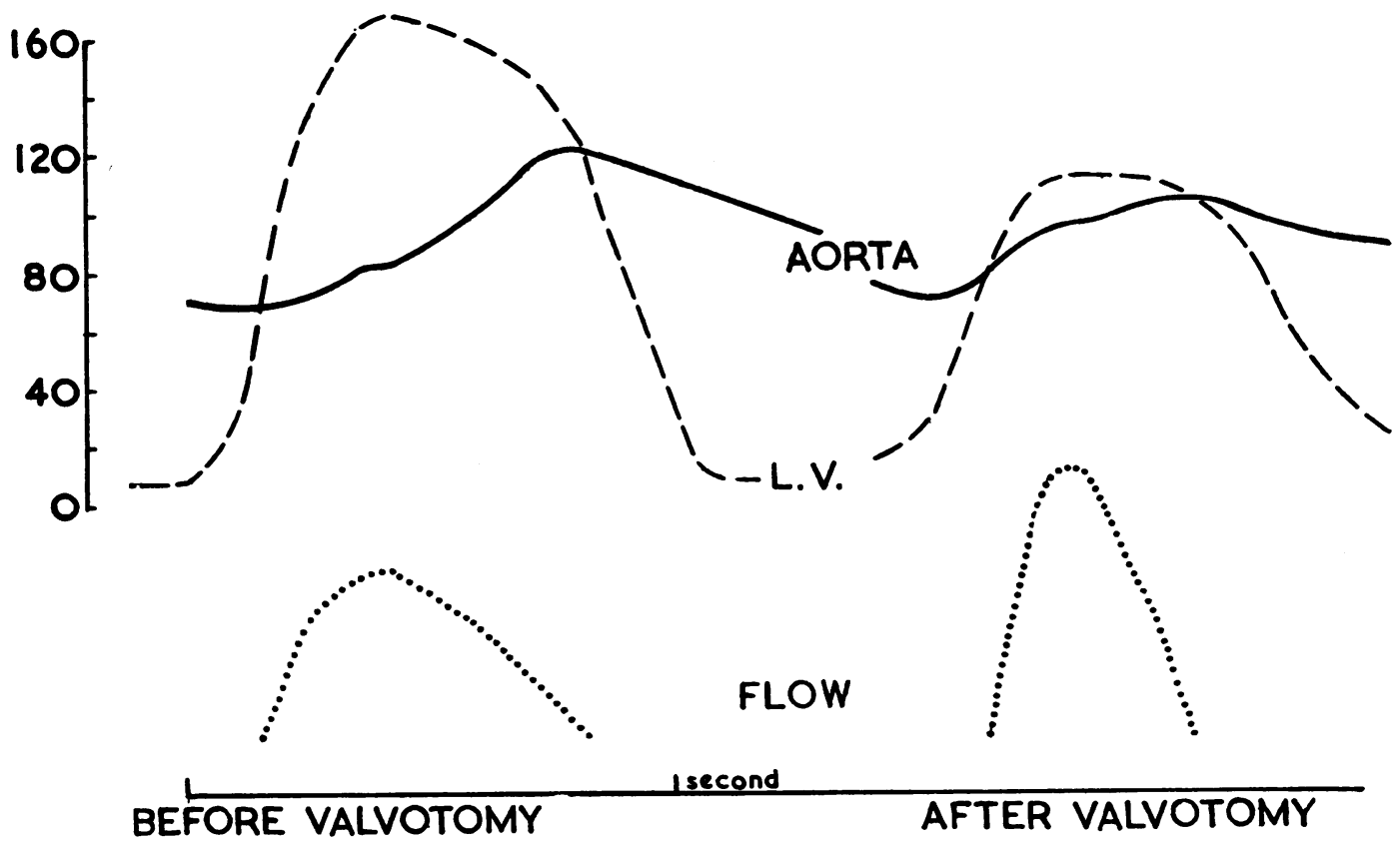

FIG. 4.-Superimposed left ventricular and aortic pressure pulses with flow curves derived from these from a case of aortic stenosis before and after aortic valvotomy. After valvotomy, there is abbreviation of the aortic upstroke time and the anacrotic notch is higher on the upstroke and less well-defined.

are considered to be good evidence in favour of the theory of Katz et al. (1927) that the notch is produced by the suction effect (Venturi) of the high-velocity blood stream.

Wiggers (1949) pointed out that the anacrotic notch is situated lower on the upstroke in severe cases of aortic stenosis than in milder cases. Katz et al. (1927) found in dogs that the left ventricular systolic peak tended to occur earlier than normal in severe aortic stenosis and this has been confirmed in man by Fleming and Gibson (1957). For this reason, combined with the delay in the aortic systolic peak, there is a long interval between maximum flow through the valve orifice and the attainment of maximum aortic pressure; a correspondingly long interval separates the anacrotic notch from the aortic peak. In a case of milder aortic stenosis or after a successful valvotomy (Fig. 4), there is a shorter interval between maximum flow and peak aortic pressure; the notch is situated nearer to the aortic peak and, therefore, higher on the upstroke. In addition, the reduced flow velocity, consequent on the increased size of the aortic orifice, produces a smaller suction effect and a less well-defined notch.

In the bisferiens type of pulse, a notch is present at or near the summit of the aortic pressure pulse and is yet of considerable depth. From the evidence presented above, the position of the notch suggests a relatively mild degree of stenosis and in the cases in which a pulsus bisferiens was found after valvotomy, there was also a satisfactory reduction in gradient. The depth of the notch, however, suggests a high flow velocity. The velocity of flow through an orifice depends, inter alia, on the size of the orifice and the volume of blood passing through it-the ventricular stroke volume in this case. In cases of atrial septal defect, the right ventricular stroke volume is often much above normal and Kjellberg et al. (1955) have emphasized the effect of the "pressure loss of velocity" on the form of the pulmonary arterial pressure pulse in this condition. In cases of atrial septal defect, a systolic gradient of $20-30 \mathrm{~mm}$. $\mathrm{Hg}$ across the pulmonary valve may be found-a gradient that is no longer present after surgical closure of the defect (Brock, personal communication). Fig. 5 illustrates a case of atrial septal defect where the peak of the pulmonary arterial pressure pulse is deformed by a well-marked notch: in this case the right ventricular stroke volume was $250 \mathrm{ml}$. It 
seems, therefore, that a high flow velocity, due to an increased stroke volume alone, is sufficient to produce a well-marked Venturi effect. A similar appearance is seen in the aortic pressure pulse in cases of persistent ductus arteriosus where the left ventricular stroke volume is abnormally large. Fig. 6 illustrates the effect of releasing a clamp on the ductus at operation: immediately the increased stroke volume and flow velocity produce a notch at the summit of the aortic pressure pulse.

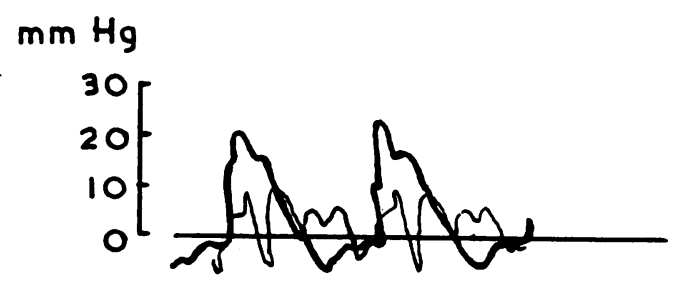

FIG. 5.-Tracing of superimposed right ventricular (thick line) and pulmonary arterial (thin line) pressure pulses from a case of atrial septal defect. The pulmonary arterial pressure pulse is deformed by a deep notch at its summit, due to the large right ventricular stroke volume $(250 \mathrm{ml}$.) and high flow-velocity.

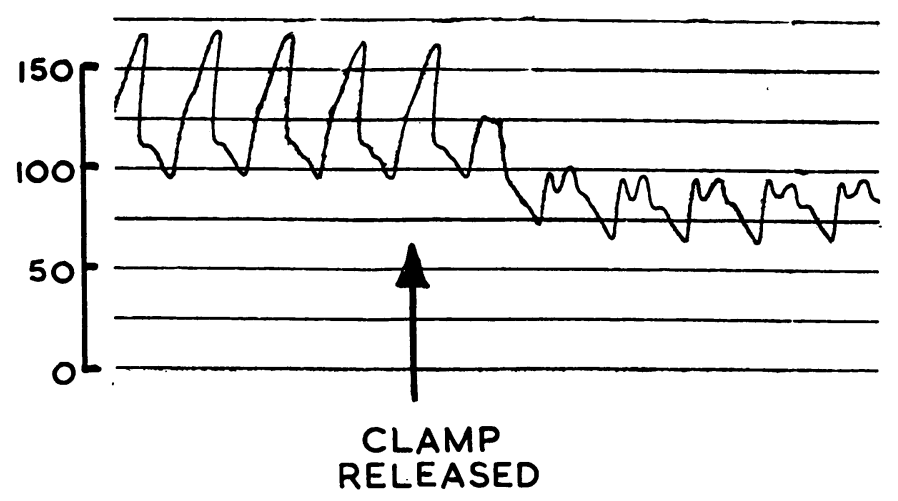

Fig. 6.-Aortic pressure pulse from a case of persistent ductus arteriosus. At the arrow a clamp on the ductus was released and a notch appeared on the summit of the pressure pulse, due to the increase in left ventricular stroke volume.

In cases with aortic regurgitation, the left ventricular stroke volume is increased by the amount of blood returning to the left ventricle in diastole. If there is, in addition, a mild degree of aortic stenosis, the flow velocity will be very high with little delay in the upstroke of the aortic pressure pulse. The notch produced by this high velocity will be situated on or near the summit of the aortic pressure pulse, which then has the form of the classical pulsus bisferiens of aortic stenosis and regurgitation (Fig. 2).

A further point of evidence against the concept of a reflected tidal wave is provided by comparison of the central and peripheral pulses in aortic valve disease. If part of the tidal wave is in fact reflected from the periphery, it should be possible to record this wave returning to the aorta in a peripheral arterial pressure pulse; this has not been done. It is considered, therefore, that the terms percussion wave and tidal wave are unacceptable, at least in this connection, and that the pulsus bisferiens does not consist of two separate waves but of a single wave split by a marked Venturi effect-the quies media of Galen. As the pulsus bisferiens indicates mild aortic stenosis with significant regurgitation, its presence is a contra-indication to aortic valvotomy. 


\section{SUMMARY}

Aortic and left ventricular pressure pulses recorded simultaneously at operation in 29 cases of aortic stenosis have been studied. A curve representing the changing gradient across the aortic valve has been derived from the superimposed pressure pulses; this curve has been used as an approximation to the curve of changing flow through the aortic orifice.

The anacrotic notch in the aortic pressure pulse is always found to be exactly synchronous with the moment of maximum flow, and it is suggested that the notch is produced by the suction effect of the high-velocity blood-stream.

In severe aortic stenosis, the position of the notch, low down on the upstroke, is shown to be related to the slow build-up of aortic pressure and the long interval between maximum flow through the aortic orifice and the aortic pressure peak.

The effect of a high flow-velocity produced by a large stroke volume alone is demonstrated by study of pulmonary arterial and aortic pressure pulses in atrial septal defect and persistent ductus arteriosus respectively. In both cases a well-defined notch is present at the summit of the pressure pulse.

The position of the notch in the pulsus bisferiens of combined aortic stenosis and regurgitation is interpreted as indicating only a slight delay in the aortic upstroke and therefore mild stenosis; its depth indicates a high flow velocity, due to the large stroke volume of aortic regurgitation. The presence of a pulsus bisferiens is, therefore, a contra-indication to aortic valvotomy.

Evidence from a comparison of central and peripheral arterial pressure pulses does not support the concept of a reflected component of the tidal wave and it is suggested that the anacrotic and bisferiens pulses do not consist of separate percussion and tidal waves, but are single waves deformed by the suction effect (Venturi effect) of a high-velocity blood stream.

All the operations were performed by Sir Russell Brock, to whom I am indebted for his co-operation in the recording of the pressure pulses.

\section{REFERENCES}

Bramwell, J. C. (1937). Lancet, 2, 239, 301, and 366.

Broadbent, W. H., and Broadbent, J. F. H. (1900). Heart Disease. 3rd ed., Baillière, Tindall \& Cox, London.

Fleming, P. R., and Gibson, R. V. (1957). Thorax, 12, 37.

Galen, C. tr. Hermannus Cruserius Campensis (1532). De Pulsibus. Simon Colinaeus. Paris.

Katz, L. N., Ralli, E. P., and Cheer, S. N. (1927). J. clin. Invest., 5, 205.

Kjellberg, S. R., Mannheimer, E., Rudhe, U., and Jonsson, B. (1955). Diagnosis of Congenital Heart Disease. The Year Book Publishers Inc. Chicago.

Mahomed, F. A. (1872). Medical Times and Gazette, 1, 128.

Marey, E-J. (1860). J. physiol. de l'homme, Paris, 3, 241.

Wiggers, C. J. (1949). Physiology in Health and Disease. 5th ed. Henry Kimpton, London.

Wright, J. L., Toscano-Barboza, E., and Brandenburg, R. O. (1956). Proc. Mayo Clin., 31, 120. 